\title{
Wearable nanocomposite kinesiology tape for distributed muscle engagement monitoring
}

\author{
Yun-An $\operatorname{Lin}^{1,2} \cdot$ m. c. schraefel ${ }^{3} \cdot$ Wei-Hung Chiang ${ }^{4} \cdot$ Kenneth J. Loh ${ }^{1,2}$ (i)
}

Received: 18 December 2020 / Accepted: 9 January 2021 / Published online: 28 January 2021

(c) The Author(s) 2021

\begin{abstract}
Sports coaches help athletes develop and improve their skills by assessing their ability to perform motion primitives that make up functional sports tasks. Sports coaching today is mostly done visually, which demands constant attention and can be imprecise. While sensors like electronic textiles and surface electromyography can measure muscle engagement, they are susceptible to movement artifacts and noise due to surface electrode issues. Therefore, the work reported here focuses on our development of self-adhesive, fabric-based sensors that can be directly affixed onto skin for monitoring skin-strains and distributed muscular engagement during functional movements. The vision is that these sensors can be readily used by sports coaches and individuals to better assess motion primitives and the execution of sports tasks. The approach integrates piezoresistive graphene nanosheet thin films with kinesiology tape (K-Tape). Because every location of the film is responsive to strains, electrodes can also be judiciously placed along the nanocomposite for distributed strain sensing. Nanocomposite or "Smart K-Tape" sensors were fabricated, and electromechanical tests were conducted to characterize their tensile, compressive, and cyclic sensing properties. Upon confirming their linearity, repeatability, stability, and high sensitivity, individuals wore Smart K-Tape sensors over different muscle groups as they performed prescribed exercise and stretching movements. The Smart K-Tapes outputted unique waveforms that revealed the speed and duration of muscular engagement through movement sequences. Furthermore, the region of muscular contraction could also be localized using each Smart K-Tape as a distributed strain sensor, which demonstrated promise as a convenient and quantitative motion primitive assessment tool relevant for sports coaching and athletic skills development.
\end{abstract}

Keywords Graphene $\cdot$ Movement $\cdot$ Muscle $\cdot$ Skin $\cdot$ Sports coaching $\cdot$ Strain sensing

\section{Introduction}

Athletes of all ages, competition levels, and sports all aim to develop their skills, improve their athletic performance, and prevent injury. Athletic skills and functional sports tasks, such as kicking a soccer ball or throwing a baseball, can be

Kenneth J. Loh

kenloh@ucsd.edu

1 Department of Structural Engineering, University of California San Diego, La Jolla, CA, USA

2 Active, Responsive, Multifunctional, and Ordered-materials Research (ARMOR) Laboratory, University of California San Diego, La Jolla, CA, USA

3 Department of Electronics and Computer Science, University of Southampton, Southampton, UK

4 Department of Chemical Engineering, National Taiwan University of Science and Technology, Taipei, Taiwan broken down into motion primitives [1]. Thus, the completion of an athletic movement sequence can be regarded as how the brain selects, combines, and executives different motion primitives [2]. In addition, many of these motion primitives are shared among different sports, so improving one's ability to perform motion primitives can result in athletic performance improvements or rapid skills development across different sports.

The role of sports coaches is to help athletes build and improve these motion primitives [3], which entails developing self-awareness and stronger brain-to-muscle connections that facilitate activating the muscles and motion primitives inherent to an athletic task [2]. Therefore, central to coaching is the assessment of athletes' strength, coordination, balance, and motor skills to guide their movements and training regime, with the end goal of maximizing their innate potential and improving performance. Today, such assessments are commonly performed by visual observations [3], which 
demands constant coach-and-athlete interactions and can be imprecise, not to mention that even the expert coach can miss or misread an athlete's movements.

For a small fraction of athletes (e.g., top professional players), sports coaching is supplemented with detailed quantitative measurements of distributed bodily movements using motion capture (mocap) [4, 5]. Mocap employs a network of synchronized cameras that can accurately quantify and record the absolute three-dimensional (3D) movements of subjects wearing retroreflective markers. However, its high costs and intricate setup makes mocap out of reach for many coaches and individuals. Even if they are used, mocap is often limited to a laboratory-like or controlled environment, because its cameras need to be stationary and cover a limited field of view. Moreover, mocap only measures discrete 3D positional changes (i.e., even when used in conjunction with accelerometers and inertial measurement units (IMU)) that inform skeletal kinematics but provides no insights about muscular engagement and force exertion. Markers worn over clothing can also be a major source of error due to its relative motion with respect to the underlying skin.

On the other hand, consumers today have access to a plethora of wearable sensors that measure various physiological parameters (e.g., step counts, heart rate, oxygen saturation, body temperature, and respiration rate) [6-9]. However, these measurements only provide a global sense of personal wellbeing and are less relevant nor specific enough for facilitating coaching and evaluating athletic performance. Electronic textiles (e-textiles) that incorporate conductive materials in fabric for direct sensing (e.g., surface electromyography (sEMG)) and actuation (e.g., functional electrical stimulation) have also been developed and used [10, 11]. While sEMG data can inform about muscle engagement, e-textile sensors are potentially susceptible to movement artifacts and greater measurement noise due to surface electrode contact effects. Therefore, an alternative is to employ ultrathin, conformable, and stretchable polymer- or nanocomposite-based sensors that can be directly affixed onto skin to monitor strains induced during different functional movements [12-14]. The measurement of vitals [15, 16], limb movement [17], joint rotations [18], and skin-strains $[19,20]$, among others, have been demonstrated. What is missing is a convenient, robust, and skin-mounted sensor that can help coaches and individuals quantitatively evaluate muscle activation and muscle synergies (or the lack thereof) during execution of motion primitives critical for enhancing athletic performance.

As a step toward this goal, this study aims to develop a self-adhering, fabric-based, wearable sensor that can be used by sports coaches to measure distributed muscle engagement involved in performing motion primitives and athletic functional movements. The proposed fabric-based sensor builds on a previous study that integrated piezoresistive graphene nanosheet (GNS) thin films with kinesiology tape (K-Tape) for skin-strain monitoring [21]. In addition to human validation studies, unique to this work is characterizing these "Smart K-Tape" for sensing both tensile and compressive strains, as well as validating the monitoring of different degrees of muscular engagement for the same range of motion and functional movement. Moreover, electrodes can be judiciously placed along a continuous nanocomposite for distributed strain sensing and for localizing contraction within a specific muscle group, which is essential for sports coaching. While Smart K-Tape cannot obtain as detailed muscular engagement information versus sEMG, they are less susceptible to noise and improper skin contact issues. This paper begins with a description of the laboratory-based experiments and human participant tests performed. The results are discussed in detail, and the paper ends with a concise summary and directions for future research.

\section{Experimental details}

\section{Smart K-Tape fabrication}

Smart K-Tape were fabricated by spray coating GNS-based thin films directly onto masked, self-adhesive, kinesiology tape substrates (Fig. 1), similar to the procedure employed in a previous study. In short, the sprayable ink was prepared by dispersing water-assisted liquid-phase exfoliated GNS in an ethyl cellulose (EC) solution by ultrasonication. A Paasche airbrush was used to manually spray coat the ethyl alcohol-based GNS-EC ink. Three layers of thin films were deposited, while a fourth layer was deposited by directly drop-casting a small amount of GNS-EC ink to enhance the thin film's uniformity and electrical conductivity. MicroRaman spectroscopy showed that the GNS-EC thin film thickness was $\sim 5$ to $15 \mu \mathrm{m}$ thick, and the GNS-EC film was
Fig. 1 Smart K-Tape fabrication process that involves direct spray coating of a graphene nanosheet dispersion is illustrated

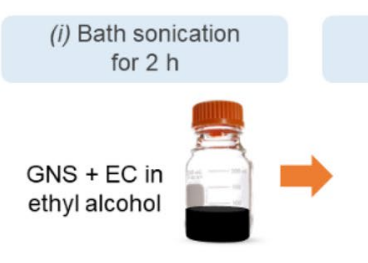

(ii) Heat and stir at $60^{\circ} \mathrm{C}$ for $12 \mathrm{~min}$

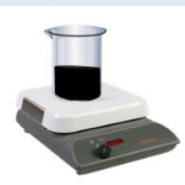

(iii) Spray-coat thin film

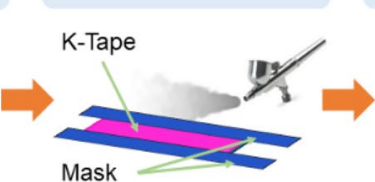

(iv) Smart K-Tape

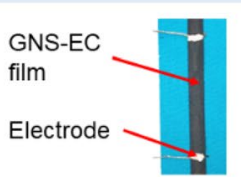


well-integrated with fabric fibers that make up the K-Tape substrate. Once the nanocomposite sensing element dried, conductive thread electrodes were sewn at the desired locations, followed by applying colloidal silver paste over the thread and film to minimize contact impedance [21].

\section{Sensing characterization}

Three sets of sensing characterization tests were performed on Smart K-Tape specimens. The first two sets of tests employed Smart K-Tape specimens fabricated with a rectangular sensing element with two electrodes, each mounted on its opposite ends, to form a two-point probe electrical resistance measurement setup, such as Fig. 2a. Load tests were conducted using a Test Resources 100R load frame, while electrical resistance was recorded using Keysight 34401A digital multimeter(s) sampling at $2 \mathrm{~Hz}$. Datalogging was digitized using the Keysight BenchVue software.

First, a three-point bending test setup was employed for characterizing both the compressive and tensile sensing performance of Smart K-Tape. A polylactic acid (PLA) beam $\left(80 \times 30 \times 3 \mathrm{~mm}^{2}\right)$ was fabricated using an Ultimaker $3+3 \mathrm{D}$-printer. A strain gage, along with a Smart K-Tape specimen, were installed adjacent to one another at midspan. Both the reference strain gage and Smart K-Tape were simultaneously interrogated by separate but synchronized multimeters. The 3D-printed PLA beam and its instrumented sensors were mounted face-up and face-down during threepoint bending to induce compressive and tensile strains, respectively. Second, Smart K-Tape specimens were subjected to tensile cyclic strains to a peak strain of $10 \%$ for 1000 cycles while recording their electrical resistance time histories. The purpose of this fatigue-like test was to characterize its repeatability and long-term sensing performance.

The final set of tests aimed to verify localized strain sensing. Longer Smart K-Tape specimens were fabricated with multiple equidistantly spaced conductive thread electrodes to form four consecutive sensing elements along each continuous GNS-EC thin film. Rectangular 3D-printed PLA mounting blocks were fabricated and attached beneath each electrode. Then, an identical bare K-Tape was affixed onto the opposite side of the mounting blocks to form the test specimen shown in Fig. 2b. Then, localized strain tests were performed by manually pulling apart two consecutive mounting blocks to selectively strain just one sensing element among the entire GNS-EC film and Smart K-Tape. The resistance of all four sensing elements were recorded simultaneously using a Keysight 34980A multifunctional switch data acquisition (DAQ) system.

\section{Human subject testing}

Human subject functional movement validation tests were conducted by affixing Smart K-Tapes onto skin and over certain major muscle groups of healthy individuals. First, demonstration of functional movement monitoring was achieved by mounting a Smart K-Tape over a subject's gluteal muscles as illustrated in Fig. 3a, while the subject performed squats. In addition to being able to capture a person's specific movements, the objective was also to measure muscular engagement. Thus, the second set of subject tests entailed mounting a Smart K-Tape over a subject's biceps (Fig. 3b) while performing biceps curls using different weights (i.e., 5, 10, 25 , and $30 \mathrm{lb}$ ). Biceps curls were done at approximately the same speed and range of motion. These human subject tests employed Smart K-Tapes, each with a single sensing element, which were interrogated using the Keysight 34980A multifunctional switch.

The last set of human subject tests was to verify distributed strain monitoring, specifically, for localizing the region of muscular contraction within the gluteal maximus (i.e., the butt) when performing different stretching movements that
Fig. 2 a A Smart K-Tape was prepared with two conductive thread and silver paste electrodes for two-point probe electrical resistance measurements. b A Smart K-Tape was instrumented with multiple electrodes along the length of the nanocomposite for linear distributed strain sensing

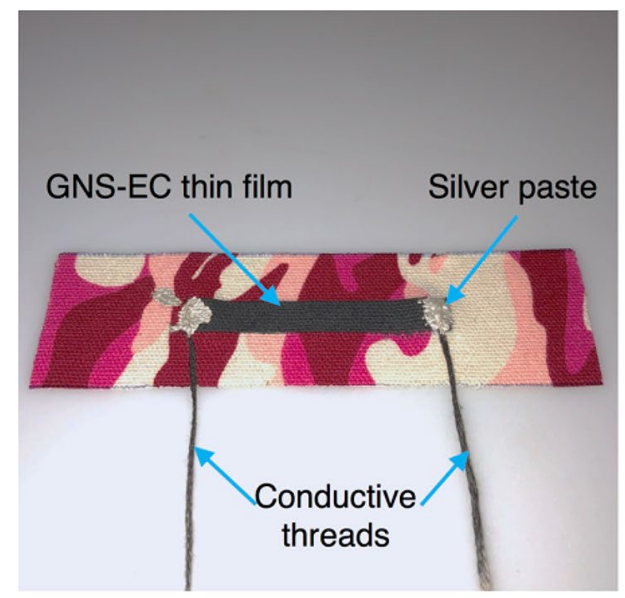

(a)

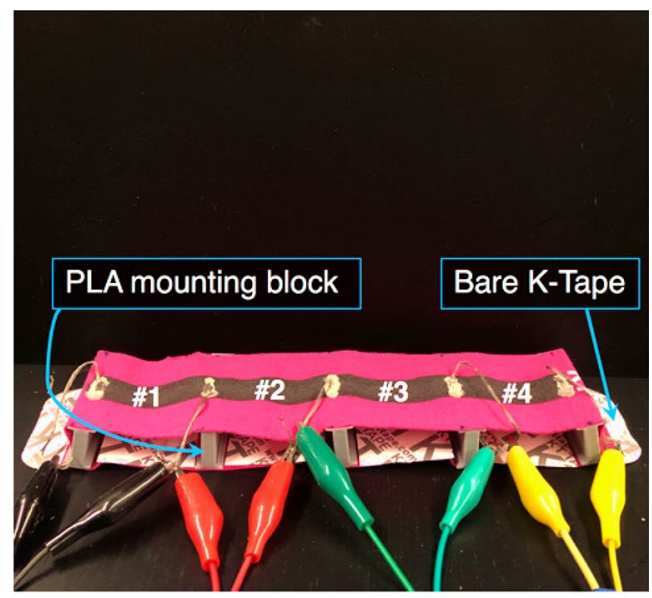

(b) 
Fig. 3 a A Smart K-Tape was mounted over the subject's gluteal muscles. b The picture shows a Smart K-Tape mounted over a subject's biceps

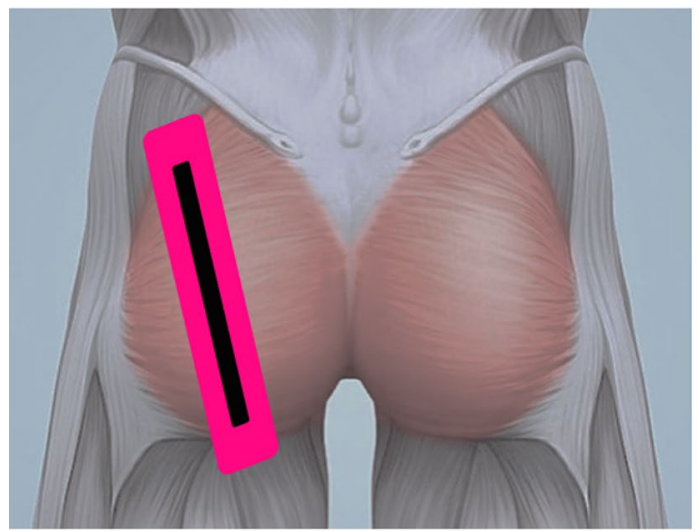

(a)

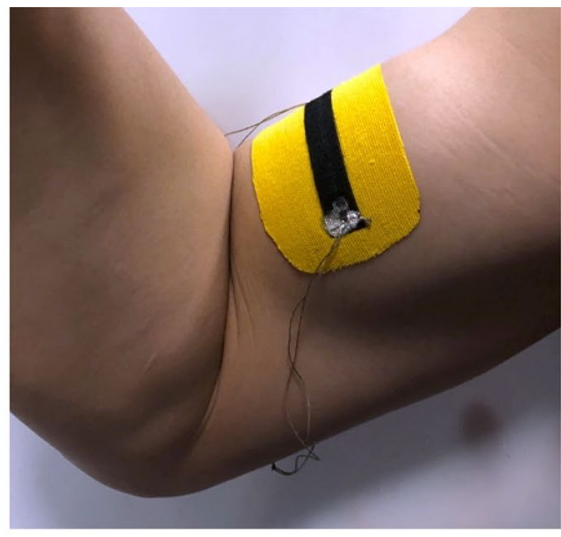

(b) activated either the upper or lower muscle fibers. Here, a Smart K-Tape with four consecutive sensing elements (similar to Fig. 2b) was affixed onto a subject's glute area, spanning the entire length of the gluteus maximus as illustrated in Fig. 3a. In one movement scheme, the upper muscle fibers were contracted by performing a leg extension, followed by a slight hip extension, and then slowly performing hip abduction while extending the leg laterally. On the other hand, activation of the lower muscle fibers was achieved with the same initial leg and hip extension but instead performing a hip adduction followed by extending the leg in the opposite direction. Similar to the laboratory-based distributed strain sensing tests, the resistance time histories of all four sensing elements were recorded simultaneously using the Keysight 34980A multifunctional switch.

\section{Results and discussion}

\section{Tensile and compressive sensing}

The tensile and compressive strain sensing properties of Smart K-Tape were characterized by conducting three-point bending load frame tests, and a representative set of results are plotted in Fig. 4. The induced tensile and compressive strains, as measured by the mounted reference strain gage, are also overlaid in each plot. Figure 4 a confirms previous findings, where the tensile sensing performance of Smart K-Tape closely follows that of the applied strain pattern during three-point bending. Furthermore, by comparing the time-synchronized resistance response against the measured strains, the strain sensitivity $(S)$ or sensor gage factor was calculated as follows:

$S=\left(\Delta R / R_{0}\right) / \Delta \varepsilon$

where $\Delta R$ is the Smart K-Tape's change in resistance with respect to its nominal resistance $\left(R_{0}\right)$ at $0 \%$ strain, and $\Delta \varepsilon$ is change in strain applied according to the reference strain gage. For this sensor tested, $S$ was $\sim 65.5$, which is $\sim 32$ times greater than the strain sensitivity of the reference strain gage (i.e., $S=2.11$ ).

Figure $4 \mathrm{~b}$ shows the compressive strain sensing response of Smart K-Tape, which is also overlaid with the measured strains. Overall, the Smart K-Tape responded to compressive strains by decreasing its resistance in tandem with greater compression applied. Like its tensile strain sensing response, these results are expected. In tension, graphene nanosheets are strained and pulled apart from one another, decreasing the number of GNS junctions for conducting electrical
Fig. 4 a A resistance time history of the three-point bending load frame tests is shown, where the Smart K-Tape and strain gage were mounted facedown, to induce tension. b A similar compression test result is plotted

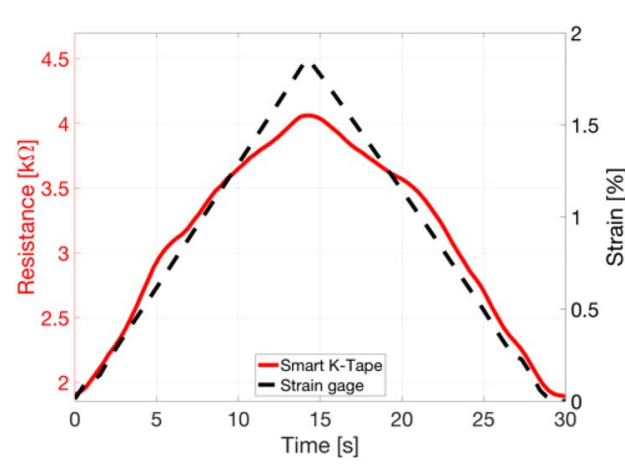

(a)

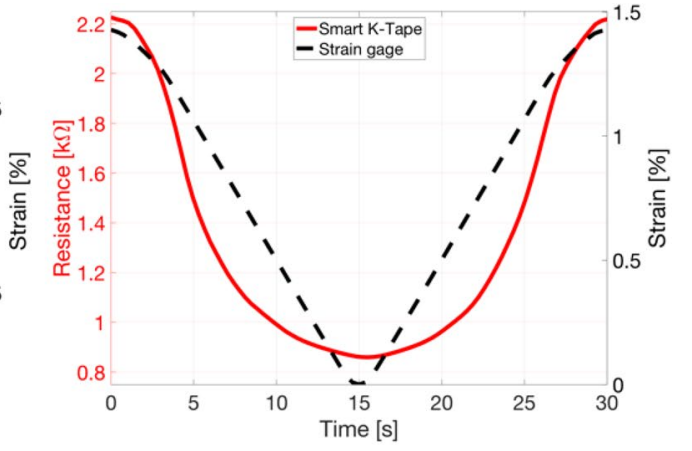

(b) 
current, thereby increasing resistance. The opposite occurs in compression, where graphene nanosheets are pushed toward one another to facilitate better electrical current conduction, thus lowering resistance. Unlike tensile strain sensing, the compression sensing response exhibited greater nonlinearity (which is not desirable), despite presenting a significantly higher $S$ of $\sim 127$. It is hypothesized that this nonlinearity may be due to how the fabric fibers of K-Tape were deforming and locally buckling. This will be investigated in more detail in future studies. Nevertheless, in practical applications of K-Tape, the fabric is pre-stretched prior to being applied onto skin. The result of pre-tensioning means that, even when movement induces compression in the skin, the entire Smart K-Tape may still be strained in only tension. As such, the sensing response will follow that of Fig. $4 \mathrm{a}$ and exhibit stronger linearity and consistent sensitivity.

\section{Cyclic electromechanical properties}

Tensile cyclic load tests were performed on Smart K-Tape specimens to assess their sensing response stability and repeatability. A representative resistance time history of a Smart K-Tape strained to $10 \%$ peak strain for 1000 cycles is plotted in Fig. 5. Closer inspection of Fig. 5 shows that the resistance at peak strain of each cycle remains fairly consistent. In addition, sensor resistance returns to its nominal resistance value, and there are no indications of baseline resistance drift, which can be an issue in other nanocomposite strain sensors. For this specimen, the average $S$ was $\sim 50.5$ and the average correlation coefficient was $\sim 0.949$. Overall, the cyclic response of Smart K-Tape exhibits strong linearity (i.e., in tension), as well as repeatable, stable, sensing properties, respectively. These results are consistent with a

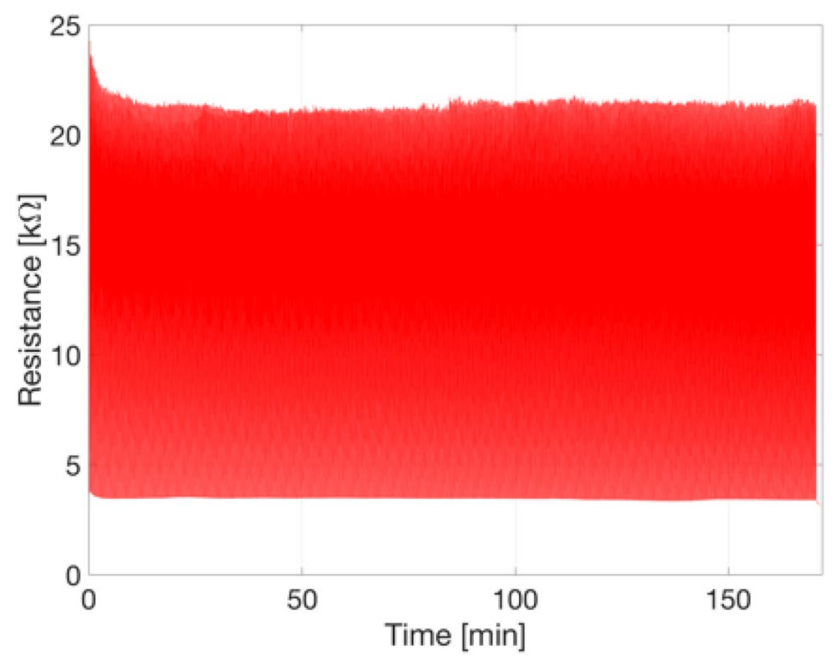

Fig. 5 A representative resistance time history of a Smart K-Tape strained to $10 \%$ peak strain for 1000 cycles is shown previous study by Lin et al. [21], while this work expanded to demonstrate favorable Smart K-Tape sensing properties at greater strain amplitudes (10\% versus 3\%) and over larger number of cycles (1000 versus 200).

\section{Distributed sensing validation}

A long Smart K-Tape strip was instrumented with five equidistantly spaced conductive thread electrodes along its length to form four consecutive thin film strain sensors. The objective was to verify that strain applied to one sensor does not affect the resistance of other sensors, considering that the four sensors interrogated different regions of the same nanocomposite. The results from after straining each sensor individually (i.e., by manually stretching the sensor in tension using its corresponding mounting blocks) while simultaneously recording the resistance of all four sensing elements is presented in Fig. 6. Figure 6 confirms that the continuous nanocomposite can be selectively interrogated, where localized strains at one region of the continuous film would not affect the resistance at other regions. Therefore, a single Smart K-Tape can be used for sensing skin-strains over different regions where it is applied.

\section{Human studies validation}

The first two sets of human tests were performed using Smart K-Tape prepared with two opposite electrodes. First, a long Smart K-Tape strip was affixed over the gluteal muscles' region, and data were collected as individuals
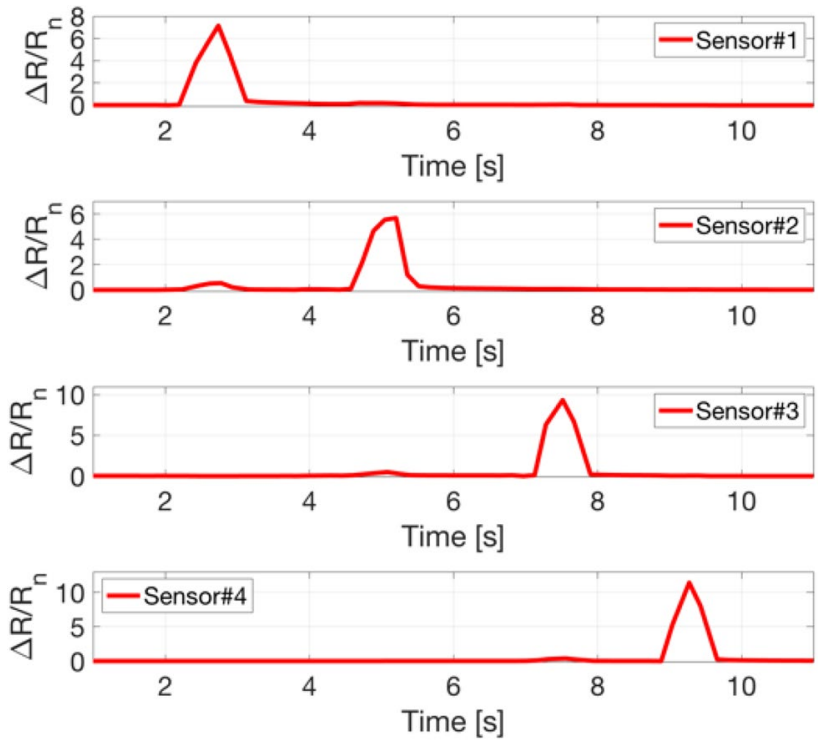

Fig. 6 Resistance time history of each sensing element in the localized strain tests is plotted. The test entailed manually pulling apart two consecutive mounting blocks to selectively strain just one sensing element at a time 


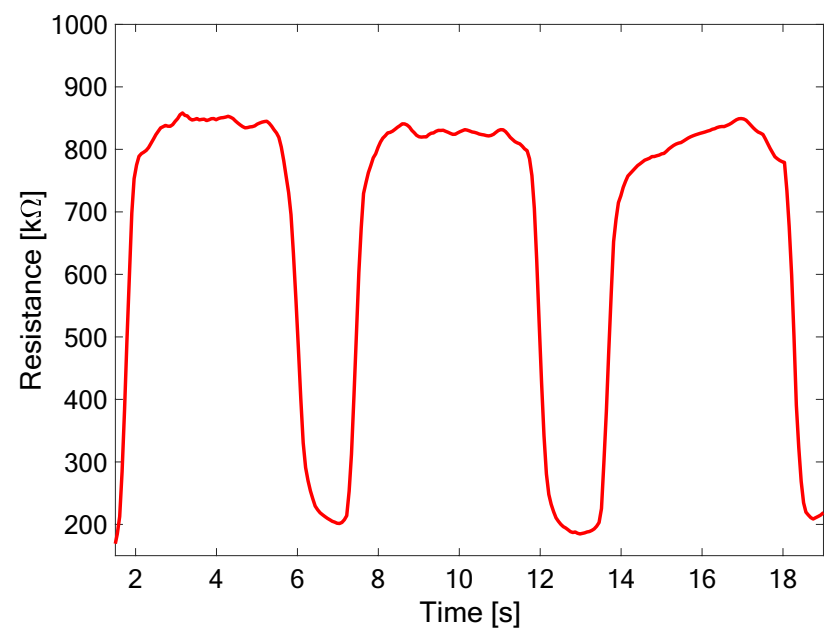

Fig. 7 Response of a Smart K-Tape affixed onto a subject's gluteal muscles while squats were performed is shown

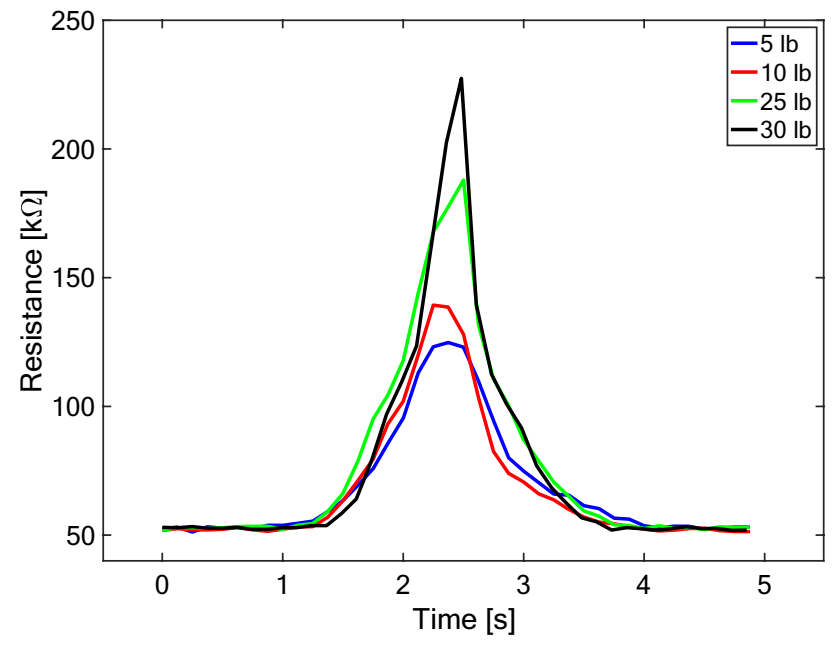

Fig. 8 Sensing response of Smart K-Tape was unique when standing biceps curls were performed using different weights (i.e., 5, 10, 25, and $30 \mathrm{lb}$ )

performed repeated squats (Fig. 3a). A representative resistance time history of the Smart K-Tape is plotted in Fig. 7. The resistance of the Smart K-Tape increased as the subject transitioned from standing to a squat position. Figure 7 shows that the resistance held stable at a high value when the subject maintained the squat position for a few seconds. The increase in resistance at the squatted position indicated tension in the gluteal muscles, which is expected. It can also be seen in Fig. 7 that sensor resistance returned to its nominal value upon returning to the standing position, before similar sensor outputs were observed when additional squats were performed. Overall, the Smart K-Tape exhibited high signalto-noise ratio and high sensitivity.
Next, the results from subjects performing bicep curls with different weights are shown in Fig. 8. Figure 8 overlays the Smart K-Tape responses collected from four standing biceps curls performed using different weights (i.e., 5, 10,25 , and $30 \mathrm{lb}$ ) but all by the same individual. Although all the biceps curls were conducted with the same range of motion, the results show that the Smart K-Tape was able to measure greater electrical resistance changes when heavier weights were lifted. The increase in peak resistance was consistent, with the $5 \mathrm{lb}$ biceps curl motion resulting in the lowest Smart K-Tape resistance change and the $30 \mathrm{lb}$ movement responding with the largest resistance change. These results are promising in that Smart K-Tape can not only be used for monitoring skin-strains and joint rotation angles but also for assessing muscular engagement of a major muscle group.

The objective of the last set of tests was to demonstrate that distributed sensing using Smart K-Tape could localize the contracted region of a major muscle group, which is critical for assessing the contraction of muscles for executing motion primitives. In addition, it is not uncommon for athletes learning a movement or rehabilitating from injury not to be contracting much of the muscle fibers available for that movement. For example, the gluteus maximus is a large muscle but with both upper and lower fibers that assist with different parts of leg extension and rotation at the hip. It is not unusual for part of that area simply not to be "firing" when an athlete attempts, for instance, to decelerate in a run or to complete an Olympic lift snatch. By helping an athlete learn how to feel and focus on these areas of muscle, they can effectively and immediately improve their strength - and often skill— by being able to bring more force to bear as soon as those fibers are activated. That activation is achieved by both appropriate movement(s) to induce a contraction as well as bringing awareness to that area. Being able to identify where along a muscle contraction or deformation may not be happening can offer valuable feedback about where to focus attention for better muscular activation.

As mentioned earlier, subjects wearing a Smart K-Tape (with multiple electrodes) were asked to perform two different stretching movements that targeted the contraction of either the upper or lower fibers of the gluteus maximus. Figure 9 plots a pair of representative results, with each corresponding to three full cycles of the movement sequence described earlier. For Fig. 9a, the hip abduction motion facilitated the contraction of the upper fibers, while the lower fibers remained fairly relaxed. The Smart K-Tape responses measured corresponding to these locations not only captured the strong contraction of the upper fibers, but they also revealed how the muscle fibers were engaged during the entire stretching sequence (i.e., the forward movement of leg extension, hip extension, and hip abduction, followed by the reverse sequence to return to the standing neutral position). 

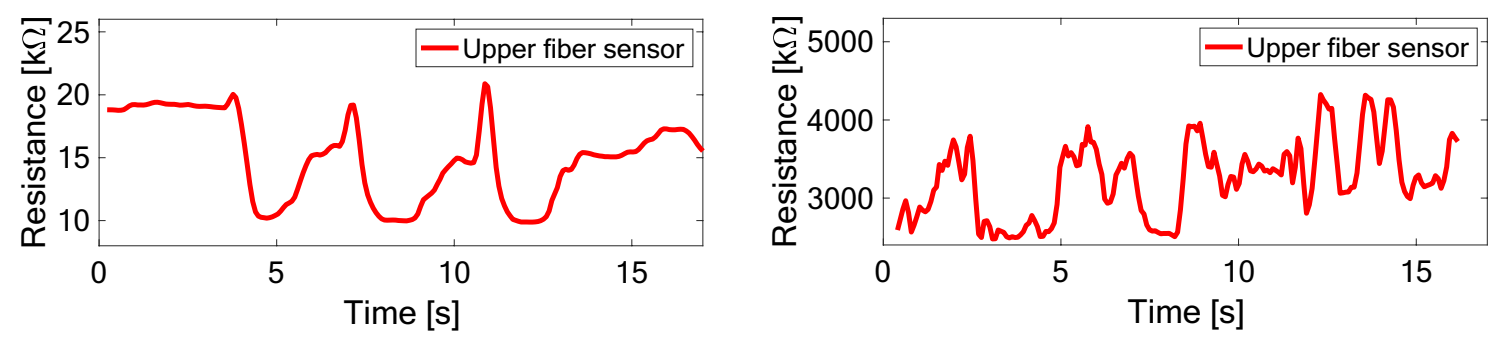

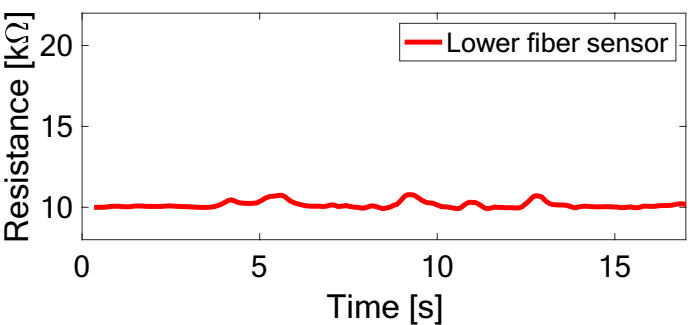

(a)

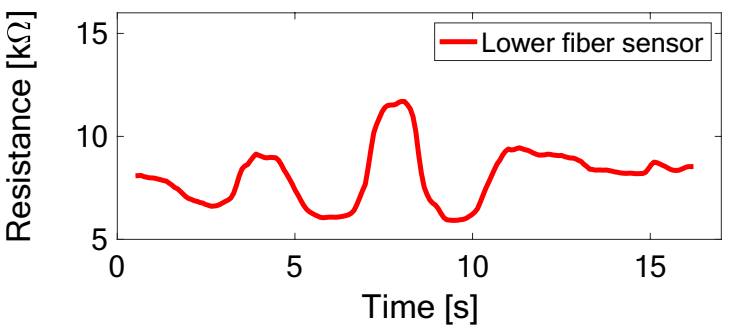

(b)

Fig. 9 a Hip abduction induced contraction in the upper fibers of the gluteal maximus, and this was captured by the Smart K-Tape. b Similarly, hip adduction contracted the lower fibers

Similar results were also observed for the three-cycle lower fibers' stretch, as is shown in Fig. 9b. In general, the Smart K-Tape portion over the lower fibers captured the hip-adduction-induced lower fiber contraction, as well as the entire stretching sequence of motion, while the sensor outputs for the upper fibers were fairly constant. It should be noted that the upper fiber Smart K-Tape response in Fig. 9b was noisier, and this was likely induced by the subject's garment. The resistance near the upper fiber portion also increased significantly (i.e., $~ 3 \mathrm{M} \Omega$ versus $\sim 20 \mathrm{k} \Omega$ from the test result in Fig. 9a); thus, it was also possible that the nanocomposite was damaged during testing. Overall, these results successfully validated the use of Smart K-Tape for distributed sensing and for localizing the general region of muscular contraction.

\section{Conclusions}

The goal of this work was to design and test a fabric-based sensor that can be used by sports coaches for assessing motion primitives and athletic functional movement. Smart K-Tapes for skin-strain and muscular engagement monitoring were fabricated. First, laboratory load frame tests confirmed their tensile and compression sensing properties. Linear tensile strain sensing was verified, but nonlinear compressive sensing response was observed. Second, a controlled distributed strain sensing test was performed using Smart K-Tape instrumented with multiple electrodes.
As expected, localized applied strains would only change the resistance of the corresponding portion of the Smart K-Tape. Next, a series of human studies was performed, where it was found that Smart K-Tape could effectively capture skin-strains induced during exercise movements (i.e., squat). In addition, biceps curl tests showed that the peak electrical resistance of Smart K-Tape increases when heavier weights were lifted, despite undergoing the same range of motion. Finally, a multi-electrode Smart K-Tape mounted over the gluteal muscles' region while different stretching movements were performed validated the monitoring of localized muscular contraction. Overall, the results demonstrated potential for Smart K-Tape to be used as a quantitative motion primitive assessment tool for sports coaching. Future studies will consider different motion primitives to evaluate their distributed sensing properties. The effects of changing environment, such as ambient temperature, humidity, and sweating, on Smart $\mathrm{K}$-Tape sensing response will also be characterized.

Acknowledgements This work was supported by the U.S. Office of Naval Research (ONR) under grant no. N00014-20-1-2329 (program manager: LCDR Dr. Joshua Swift; principal investigator: Prof. Kenneth J. Loh). Partial support was provided by Sony. Ms. Yun-An Lin was also partially supported by the J. Yang Foundation Scholarship. The authors gratefully acknowledge the collaboration of Prof. Eric Hekler from the University of California San Diego, LCDR Dr. John J. Fraser, Dr. Pinata Sessoms, and Dr. Amy Silder from the Naval Health Research Center, and Dr. Eric Cobb from Z-Health. All opinions expressed in this manuscript are the authors' and do not necessarily 
reflect the policies and views of the Department of Defense, U.S. Navy, University of California, or Sony.

Data availability Data are available upon request.

\section{Compliance with ethical standards}

Conflict of interest The authors declare no conflicts of interest.

Open access This article is licensed under a Creative Commons Attribution 4.0 International License, which permits use, sharing, adaptation, distribution and reproduction in any medium or format, as long as you give appropriate credit to the original author(s) and the source, provide a link to the Creative Commons licence, and indicate if changes were made. The images or other third party material in this article are included in the article's Creative Commons licence, unless indicated otherwise in a credit line to the material. If material is not included in the article's Creative Commons licence and your intended use is not permitted by statutory regulation or exceeds the permitted use, you will need to obtain permission directly from the copyright holder. To view a copy of this licence, visit http://creativecommons.org/licenses/by/4.0/.

\section{References}

1. A.E. Hibbs, K.G. Thompson, D. French, A. Wrigley, I. Spears, Sports Med. 38, 995-1008 (2008)

2. K. Zhao, Z. Zhang, H. Wen, Z. Wang, J. Wu, J. Healthc. Eng. 2019(8), 1-9 (2019)

3. R.L. Jones, The Sports Coach as Educator: Re-Conceptualizing Sports Coaching (Routledge, Abingdon, Oxon, 2006).

4. O. Mirabella, A. Raucea, F. Fisichella and L. Gentile, in 4th International Conference on Human System Interactions, HSI 2011, IEEE Xplore, Yokohama, Japan 2011.
5. E.V.D. Kruk, M.M. Reijne, Biomech Motor Control 18(6), 806$819(2018)$

6. G.M.N. Islam, A. Ali, S. Collie, Cellulose 27(11), 6103-6131 (2020)

7. G. Aroganam, N. Manivannan, D. Harrison, Sensors 19(9), 1983 (2019)

8. Y. Adesida, E. Papi, A.H. McGregor, Sensors 19(7), 1597 (2019)

9. Y. Gu, T. Zhang, H. Chen, F. Wang, Y. Pu, C. Gao, S. Li, Nanoscale Res. Lett. 14(1), 1-15 (2019)

10. K. Yang, B. Isaia, L.J.E. Brown, S. Beeby, Sensors 19(20), 4463 (2019)

11. K. Yang, K. Meadmore, C. Freeman, N. Grabham, A.-M. Hughes, Y. Wei, R. Torah, M. Glanc-Gostkiewicz, S. Beeby, J. Tudor, Sensors 18(8), 2410 (2018)

12. J. Chen, Q. Yu, X. Cui, M. Dong, J. Zhang, C. Wang, J. Fan, Y. Zhu, Z. Guo, J. Mater. Chem. C 7(38), 11710-11730 (2019)

13. W.A.D.M. Jayathilaka, K. Qi, Y. Qin, A. Chinnappan, W. SerranoGarcía, C. Baskar, H. Wang, J. He, S. Cui, S.W. Thomas, S. Ramakrishna, Adv. Mater. 31(7), 1-21 (2019)

14. Y. Lu, M.C. Biswas, Z. Guo, J.-W. Jeon, E.K. Wujcik, Biosens. Bioelectron. 123(1), 167-177 (2019)

15. L. Wang, K.J. Loh, W.-H. Chiang, K. Manna, Nanotechnology 29(10), 105503 (2018)

16. J. Ramírez, D. Rodriquez, A.D. Urbina, A.M. Cardenas, D.J. Lipomi, ACS Appl. Nano Mater. 2(4), 2222-2229 (2019)

17. X. Yue, Y. Jia, X. Wang, K. Zhou, W. Zhai, G. Zheng, K. Dai, L. Mi, C. Liu, C. Shen, Compos. Sci. Technol. 189(22), 108038 (2020)

18. S. Sun, L. Guo, X. Chang, Y. Liu, S. Niu, Y. Lei, T. Liu, X. Hu, J. Mater. Sci. 54, 7048-7061 (2019)

19. J. Lee, S. Kim, J. Lee, D. Yang, B.C. Park, S. Ryu, I. Park, Nanoscale 6, 11932-11939 (2014)

20. L. Wang, K.J. Loh, Smart Mater. Struct. 26(5), 055018 (2017)

21. Y.A. Lin, Y. Zhao, L. Wang, Y. Park, Y.J. Yeh, W.H. Chiang and K. J. L. Loh, Adv. Mater. Technol. (2021). 